TẠP CHÍ KHOA HỌC ĐẠI HỌC TÂN TRÀO

ISSN: $2354-1431$

http://tckh.daihoctantrao.edu.vn/

\title{
Nâng cao hiệu quả hoạt động nghiên cứu khoa học của sinh viên Trường Đại học Tân Trào
}

\author{
Lê Thị Thu Hàa* \\ ${ }^{a}$ Trưòng Đại học Tân Trào \\ *Email: lethithuhasptq@gmail.com
}

\section{Thông tin bài viết}

Ngày nhận bài:

20/12/2017

Ngày duyệt đăng:

$10 / 3 / 2018$

\section{Tù khoá:}

Khoa hoc; nghiên cúu khoa hoc; tụ nghiên cúu; phuong pháp nghiên cứu khoa hoc.

\begin{abstract}
Tóm tắt
Nghiên cứu khoa học được xác định là một trong những nhiệm vụ trọng tâm của Trường Đại học Tân Trào, chính vì lẽ đó, trong những năm qua, nhà trường luôn khuyến khích và tạo mọi điều kiện thuận lợi cho cán bộ, giảng viên, sinh viên tham gia nghiên cứu khoa học. Tuy nhiên, số lượng sinh viên tham gia nghiên cứu khoa học còn ít, chất lượng các công trình khoa học còn nhiều hạn chế. Trong bài biết này, tác giả tập trung vào nghiên cứu thực trạng hoạt động nghiên cứu khoa học của sinh viên Trường Đại học Tân Trào, trên cơ sở đó đề xuất một số giải pháp nâng cao hiệu quả hoạt động nghiên cứu khoa học của sinh viên.
\end{abstract}

\section{1. Đặt vấn đề}

Cùng với hoạt động học tập, nghiên cứu khoa học luôn được coi là một nhiệm vụ quan trọng của sinh viên trong quá trình đào tạo tại các trường đại học, cao đẳng. Điều 28 Luật Giáo dục Đại học năm 2012 quy định một trong các nhiệm vụ và quyền hạn của các cơ sở giáo dục đại học là "Triển khai hoạt động đào tạo, khoa học và công nghệ, hợp tác quốc tế, bảo đảm chất lượng giáo dục đại học". Khoản 2 , điều 39 của luật này cũng chỉ rõ mục tiêu hoạt động khoa học và công nghệ là "Hình thành và phát triển năng lực nghiên cứu khoa học cho người học; phát hiện và bồi dưỡng nhân tài, đáp ứng yêu cầu đào tạo nhân lực trình độ cao". Ý thức được mục tiêu và nhiệm vụ đó, nghiên cứu khoa học trong sinh viên tại các trường đại học, cao đẳng luôn được chú trọng và khuyến khích phát triển. Bằng chứng là trong những năm gần đây, số lượng sinh viên tham gia và nhận được các giải thưởng như "Tài năng Khoa học trẻ Việt Nam", giải thưởng "Sinh viên nghiên cứu khoa học" do Bộ Giáo dục và Đào tạotổ chức ngày càng tăng, nhiều công trình nghiên cứu có chất lượng cao, có giá trị khoa học và thực tiễn lớn. Tuy nhiên, hoạt động nghiên cứu khoa học đã thật sự thu hút, lan tỏa sâu rộng hay chỉ dừng lại ở một bộ phận sinh viên? Sinh viên gặp những khó khăn, trở ngại gì trong quá trình nghiên cứu khoa học? Làm thế nào để nâng cao hiệu quả hoạt động nghiên cứu khoa học trong sinh viên? Trong bài viết này, tác giả mong muốn chia sẻ một số quan điểm, nhận định của mình về hoạt động nghiên cứu khoa học của sinh viên trường Đại học Tân Trào và đề xuất một số giải pháp góp phần nâng cao hiệu quả hoạt động này.

\section{Nội dung nghiên cứu}

\subsection{Một số vấn đề lý luận}

\subsubsection{Khoa hoc}

Khoa học (science) là toàn bộ hoạt động có hệ thống nhằm xây dựng và tổ chức kiến thức dưới hình thức những lời giải thích và tiên đoán có thể kiểm tra được về vũ trụ. Khoa học vừa là một hình thái ý thức xã hội, vừa là một dạng hoạt động, một công cụ nhận thức, nó bao gồm hệ thống tri thức về tự nhiên, xã hội và tư duy, được tích luỹ trong quá trình nhận thức trên cơ sở thực tiễn, được thể hiện bằng những khái niệm, phán đoán, học thuyết [6]. 
Khoa học là hệ thống những tri thức về tự nhiên, xã hội và tư duy, về những quy luật phát triển khách quan của tự nhiên, xã hội và tư duy. Hệ thống tri thức này được hình thành trong lịch sử và không ngừng phát triển trên cơ sở thực tiễn xã hội $[4,15]$.

Khoa học là hình thái ý thức xã hội phản ánh thế giới khách quan, bao gồm hệ thống chân lý của thế giới quanh ta. Hệ thống này được biểu đạt dưới dạng các khái niệm, các quy luật, các học thuyết lý thuyết, các phạm trù, giả thuyết...[2, 11$]$.

Khoa học là tổng hợp các tri thức về tự nhiên và xã hội tích lũy trong quá trình lịch sử hướng đến mục đích cơ bản của nó là xây dựng lý luận để giải thích và tiên đoán các hiện tượng nhằm thực hiện chức năng xã hội của nó là phục vụ thực tiễn xã hội $[3,3]$.

Như vậy, có thể hiểu khoa học là một hình thái ý thức xã hội, là sản phẩm của quá trình nhận thức của loài người, nó bao gồm hệ thống tri thức về tự nhiên, xã hội, tư duy, về những qui luật phát triển khách quan của chúng.

\subsubsection{Nghiên cúu khoa học}

Nghiên cứu khoa học là một dạng hoạt động đặc biệt của con người. Đây là một hoạt động có mục đích, có kế hoạch, được tổ chức chặt chẽ của một đội ngũ các nhà khoa học với những phẩm chất đặc biệt, được đào tạo ở trình độ cao, là hoạt động nhận thức thế giới khách quan, là quá trình phát hiện chân lý và vận dụng chúng vào cuộc sống [4, 21].

Nghiên cứu khoa học là sự phát hiện bản chất sự vật, phát triển nhận thức khoa học về thế giới; hoặc là sáng tạo phương pháp mới và phương tiện kỹ thuật mới để làm biến đổi sự vật phục vụ cho mục tiêu hoạt động của con người [1, 35].

Nghiên cứu khoa học là quá trình nhận thức chân lý khoa học, một hoạt động trí tuệ đặc thù bằng các phương pháp nghiên cứu nhất định để tìm kiếm, chỉ ra một cách chính xác và có mục đích những điều mà con người chưa biết đến (hoặc chưa biết đầy đủ), tức là tạo ra sản phẩm mới dưới dạng tri thức mới, có giá trị mới về nhận thức hoặc phương pháp [2, 18-19].

Nghiên cứu khoa học là một quá trình vận dụng các ý tưởng, nguyên lý và sử dụng các phương pháp khoa học, phương pháp tư duy để tìm tòi, khám phá các khái niệm, hiện tượng và sự vật mới để phát hiện quy luật tự nhiên và xã hội nhằm giải quyết mâu thuẫn nhận thức và hoạt động thực tiễn để sáng tạo các giải pháp tác động trở lại sự vật, hiện tượng góp phần cải thiện cuộc sống và lao động sản xuất $[3,8]$.
Như vậy, có thể hiểu: Nghiên cứu khoa học là một hoạt động có mục đích, có kế hoạch được tổ chức chặt chẽ của đội ngũ các nhà khoa học, là hoạt động nhận thức thế giới khách quan, là quá trình phát hiện chân lí và vận dụng chúng vào đời sống.

Nghiên cứu khoa học có cấu trúc phức tạp, gồm nhiều yếu tố: xây dựng các nhiệm vụ nhận thức; nghiên cứu những phương pháp và tri thức đã có trong lĩnh vực đang nghiên cứu; đưa ra và phân tích lí thuyết những giả thuyết; phân tích và khái quát hoá những kết quả đã nhận thức được; kiểm tra các giả thuyết có được trên cơ sở tổng hợp toàn bộ các sự kiện; xây dựng các lí thuyết và hình thành những quy luật; nghiên cứu những dự báo khoa học...

Nghiên cứu khoa học bao gồm:

+ Nghiên cứu hàn lâm: nghiên cứu nhằm trả lời các câu hỏi về bản chất lý thuyết, hay nói cách khác là xây dựng và kiểm định các lý thuyết khoa học. Các lý thuyết khoa học này đóng góp vào kho tàng tri thức khoa học để giải thích và dự báo các hiện tượng khoa học.

+ Nghiên cứu ứng dụng: nghiên cứu nhằm áp dụng các thành tựu khoa học (lý thuyết khoa học) vào các lĩnh vực cụ thể trong cuộc sống.

2.1.3. Ý nghĩa của nghiên cứu khoa học đối với sinh viên

Nghiên cứu khoa học là hoạt động trí tuệ giúp sinh viên vận dụng phương pháp luận và phương pháp nghiên cứu khoa học trong học tập và trong thực tiễn, trong đó sinh viên bước đầu vận dụng một cách tổng hợp những tri thức đã học để tiến hành hoạt động nhận thức có tính chất nghiên cứu, bước đầu góp phần giải quyết những vấn đề khoa học do thực tiễn cuộc sống và nghề nghiệp đặt ra để từ đó có thể đào sâu, mở rộng và hoàn thiện vốn hiểu biết của mình.

Để thực hiện được một công trình nghiên cứu khoa học đòi hỏi người học phải hiểu về các phương pháp nghiên cứu khoa học, các bước, các giai đoạn tiến hành công trình nghiên cứu, biết tìm kiếm tài liệu, chọn lọc, phân loại tài liệu, xử lý số liệu, trình bày nội dung nghiên cứu một cách có hệ thống, logic. Nhờ vậy mà người học tích lũy thêm nhiều kiến thức, kinh nghiệm bổ ích.

Nghiên cứu khoa học hình thành ở sinh viên các kỹ năng cần thiết như: kỹ năng làm việc nhóm (trao đổi với bạn bè, thấy cô), kỹ năng tra cứu các thông tin trên mạng, kỹ năng quản lý thời gian, kỹ năng lập kế 
hoạch, kỹ năng thuyết trình, phản biện, kỹ năng tự học, tự nghiên cứu...

Mặt khác, thông qua hoạt động nghiên cứu khoa học, sinh viên hình thành cho bản thân các phẩm chất cần thiết của nhà nghiên cứu như: độc lập, sáng tạo, kiên nhẫn, tư duy phản biện, bồi dưỡng lòng say mê khoa học, hứng thú học tập, rèn bản lĩnh và sự tự tin...

Điều quan trọng là tham gia hoạt động nghiên cứu khoa học khi còn là sinh viên sẽ tạo điều kiện thuận lợi cho người học sau khi ra trường, tiếp tục học cao lên, mở rộng, đào sâu, hình thành các ý tưởng mới cho các đề tài, luận văn, luận án sau này.

\subsection{Thục trạng hoạt động nghiên cúu khoa học của} sinh viên Truò̀ng Đại học Tân Trào

Trong những năm gần đây, trường Đại học Tân Trào đã thường xuyên tổ chức các hoạt động khoa học, thu hút được nhiều cán bộ, giảng viên, sinh viên tham gia. Bên cạnh nhiệm vụ khoa học bắt buộc hàng năm như làm đề tài nghiên cứu khoa học cấp trường, viết bài báo khoa học, viết tài liệu tham khảo, giáo trình môn học... nhiều giảng viên còn tham gia đề tài khoa học cấp Bộ, cấp tỉnh, nhiều bài báo được đăng trên các tạp chí có uy tín trong nước và quốc tế. Bên cạnh đó, nhà trường còn tổ chức các hoạt động khoa học như: hội thảo, tập huấn, ngoại khóa chuyên môn...thu hút được đông đảo cán bộ, giảng viên, sinh viên tham gia. Có thể kể đến một số chương trình như: Hội thảo khoa học quốc tế "Văn hóa và ngôn ngữ các dân tộc trong sự giao thoa giữa các quốc gia Đông Nam Á"; Hội thảo khoa học "Chương trình đào tạo giáo viên mầm non, tiểu học, THCS do trường Đại học Tân Trào đào tạo giai đoạn 2006 - 2016 từ góc nhìn thực tiễn"; Chương trình ngoại khoá chuyên môn "Đổi mới phương pháp dạy học theo hướng tích hợp liên môn ở THCS hiện nay"; Hội thảo khoa học "Vật lý hiện đại và ứng dụng"; Chương trình "Ngày hội tuổi trẻ - Trao ý tưởng, đổi niềm vui"; Hội thảo "Văn học Tuyên Quang thời kỳ đổi mới”... Bên cạnh đó, sinh viên hệ cao đẳng năm thứ 2 và hệ đại học năm thứ 3 ngành sư phạm đều phải làm bài tập nghiên cứu Tâm lý - Giáo dục, đây là một nhiệm vụ mà sinh viên phải thực hiện khi đi kiến tập sư phạm. Sinh viên năm cuối hệ cao đẳng, đại học được lựa chọn để thực hiện khóa luận tốt nghiệp.

Năm học 2016 - 2017 có 10 nhóm sinh viên tham gia làm đề tài nghiên cứu khoa học cấp trường (trong đó 3 đề tài được Hội đồng đánh giá xếp loại $\mathrm{A}, 5$ đề tài xếp loại B, 2 đề tài xếp loại C); Năm học 2015 -
2016 có 32 sinh viên ngành Cao đẳng Quản lý đất đai bảo vệ thành công khóa luận tốt nghiệp; Năm học 2016-2017 có 08 sinh viên ngành cao đẳng Kế toán tổng hợp và 24 sinh viên ngành Quản lý đất đai hoàn thành khóa luận tốt nghiệp. Năm học 2017 - 2018 có 10 nhóm sinh viên đăng ký làm đề tài nghiên cứu khoa học.

Qua biên bản nhận xét của Hội đồng nghiệm thu đề tài nghiên cứu khoa học và Hội đồng chấm khóa luận tốt nghiệp thấy rằng: sinh viên bước đầu đã có hứng thú với hoạt động nghiên cứu khoa học, tuy nhiên các em chưa có kỹ năng nghiên cứu (từ việc lựa chọn đề tài, xây dựng đề cương, kế hoạch nghiên cứu đến việc sử dụng các phương pháp nghiên cứu và trình bày công trình nghiên cứu), nhiều sinh viên thiếu chủ động, còn trông chờ nhiều vào giáo viên hướng dẫn, kết quả nghiên cứu còn hạn chế.

Kết quả nghiên cứu về thực trạng hoạt động nghiên cứu khoa học của sinh viên trong nhà trường qua phiếu điều tra cụ thể như sau:

2.2.1. Nhận thức của sinh viên về tầm quan trọng của hoạt động nghiên cúu khoa học

Nhận thức đúng đắn về tầm quan trọng của hoạt động nghiên cứu khoa học ảnh hưởng rất lớn đến chất lượng công tác nghiên cứu khoa học của sinh viên. Kết quả khảo sát cho thấy không có sinh viên nào cho rằng hoạt động nghiên cứu khoa học là rất quan trọng, có $12 \%$ ý kiến cho rằng quan trọng. Trong khi đó số sinh viên cho rằng hoạt động này ít quan trọng là $57 \%$, không quan trọng là $15 \%$ và vẫn còn tới $16 \%$ sinh viên chưa xác định rõ thế nào là hoạt động nghiên cứu khoa học nên vẫn chưa đánh giá được tầm quan trọng của nó. Từ kết quả trên cho thấy sinh viên trường Đại học Tân Trào hiện nay chưa thật sự nhận thức được tầm quan trọng của hoạt động nghiên cứu khoa học trong quá trình học tập, do đó chưa có sự đầu tư thích đáng cũng như chưa tích cực tham gia vào hoạt động này.

2.2.2. Nhận thức của sinh viên về vai trò, tác dụng của nghiên cúu khoa học

Khi được hỏi: “Theo bạn, nghiên cứu khoa học có vai trò, tác dụng như thế nào đối với sinh viên?", đa số sinh viên cho rằng nghiên cứu khoa học có vai trò quan trọng giúp người học phát triển tư duy độc lập, sáng tạo (82\%), nâng cao năng lực tự học, tự nghiên cứu (78\%), hình thành kỹ năng làm việc khoa học (77\%). Ngoài ra, còn giúp người học hình thành năng lực hợp tác, gắn lý thuyết với thực hành, bồi dưỡng lòng say mê, hứng thú 
học tập, rèn luyện tính kiên trì, bền bỉ...Tuy nhiên, vẫn còn bộ phận không nhỏ sinh viên chưa có nhận thức đúng đắn, các em cho rằng: nhiệm vụ cơ bản của sinh viên là học tập còn nghiên cứu khoa học là nhiệm vụ của thầy cô giáo và của một số sinh viên khá, giỏi; nghiên cứu khoa học mất nhiều thời gian, ảnh hưởng đến việc học tập; nhiều công trình nghiên cứu xong rồi để đấy không được ứng dụng vào thực tiễn nên lãng phì thời gian, công sức...Như vậy, có thể nhận thấy không phải sinh viên nào cũng nhận thức đúng về vai trò, tác dụng của hoạt động nghiên cứu khoa học.

2.2.3. Các hình thức tham gia nghiên cứu khoa học của sinh viên

Để đẩy mạnh hoạt động nghiên cứu khoa học của sinh viên, trường Đại học Tân Trào đã chủ động đưa hoạt động này vào trong chương trình khung, kế hoạch giảng dạy hàng năm. Các hình thức nghiên cứu khoa học được nhà trường tổ chức như: Thảo luận tình huống trên lớp, làm đề tài tiểu luận môn học, làm bài tập nghiên cứu Tâm lý - Giáo dục, tổ chức hội thảo cấp Khoa, cấp trường, cấp khu vực, sinh viên làm đề tài nghiên cứu khoa học cấp trường, viết báo, Tổ chức các hoạt động ngoại khóa, Cuộc thi sáng tạo kỹ thuật trẻ, khóa luận tốt nghiệp... nhằm thu hút sinh viên tham gia nghiên cứu khoa học, khích lệ sự sáng tạo và năng động trong bước đầu làm quen với nghiên cứu.

Theo kết quả khảo sát cho thấy hoạt động nghiên cứu khoa học của sinh viên hiện nay chủ yếu ở các hình thức đơn giản, mang tính bắt buộc như là thực hiện các bài tập tiểu luận do giảng viên giao cho hoặc thực hiện các bài báo cáo chuyên đề, khóa luận 7\% (chủ yếu là sinh viên năm 3, năm 4); thực hiện Bài tập nghiên cứu Tâm lý - Giáo dục (bắt buộc 100\% sinh viên ngành sư phạm phải tham gia). Về các hoạt động mang tính tự giác, đòi hỏi sự khám phá, tìm tòi nghiên cứu sinh viên vẫn chưa tham gia nhiều, chưa thật sự quan tâm, như việc tham gia các hội thảo khoa học do khoa hoặc trường tổ chức chỉ chiếm $21 \%$. Một số sinh viên tham gia đề tài nghiên cứu khoa học cấp trường (năm học 2015 - 2016 có 10 nhóm sinh viên tham gia). Bên cạnh đó rất nhiều sinh viên chưa bao giờ tham gia các hoạt động nghiên cứu khoa học $63 \%$ (chủ yếu là sinh viên năm 1 , năm 2). Điều này cho thấy hoạt động nghiên cứu khoa học chưa phổ biến, chưa thu hút được sinh viên. Đây không phải là thực trạng riêng ở trường đại học Tân Trào, mà là thực trạng chung, là vấn đề lớn của rất nhiều trường đại học hiện nay trong cả nước.

2.2.4. Nguyên nhân sinh viên chua tích cực tham gia hoạt động nghiên cứn khoa học
Hoạt động nghiên cứu khoa học của sinh viên đã được đưa vào trong chương trình dạy và học của nhà trường, tuy nhiên trong thực tế hoạt động này chưa được lan tỏa sâu rộng, số lượng sinh viên tham gia hoạt động khoa học còn ít, chất lượng các công trình khoa học còn hạn chế. Vậy những nguyên nhân nào dẫn đến tồn tại, hạn chế nêu trên? Có thể kể đến các nguyên nhân sau:

Nghiên cứu khoa học thực sự là công việc khó. Đây là một dạng lao động trí tuệ đặc biệt, là quá trình tìm kiếm và khám phá những điều chưa biết, tìm ra những cái mới và ứng dụng chúng trong đời sống thực tiễn. Trong quá trình học tập ở nhà trường, sinh viên mới chỉ được trang bị một số kiến thức cơ bản về khoa học và nghiên cứu khoa học, các bước tiến hành một công trình nghiên cứu khoa học, chưa có kỹ năng sử dụng các phương pháp nghiên cứu cụ thể vì vậy thiếu kiến thức, thiếu kinh nghiệm thực tế. Là một giảng viên đang trực tiếp giảng dạy học phần Phương pháp nghiên cứu khoa học giáo dục cho sinh viên, tôi nhận thấy đây là môn học khó, với thời lượng 30 tiết lên lớp, người học rất khó khăn để nắm được kiến thức lý thuyết cơ bản, việc vận dụng lý thuyết để thực hành, luyện tập hình thành các kỹ năng là chưa đạt yêu cầu, mục tiêu môn học. Thực tế từ việc hướng dẫn sinh viên năm thứ 2, thứ 3 làm bài tập nghiên cứu Tâm lý - Giáo dục, sinh viên hệ đại học đã được nghiên cứu học phần Phương pháp nghiên cứu khoa học giáo dục, còn sinh viên hệ cao đẳng chưa được tiếp cận với môn học. Khó khăn này dẫn đến nhiều sinh viên kết quả nghiên cứu chưa đạt yêu cầu. Một số sinh viên thậm chí không biết đặt tên đề tài, xác định sai mục đích, nhiệm vụ, đối tượng nghiên cứu, không biết sử dụng các phương pháp nghiên cứu và trình bày vấn đề nghiên cứu lộn xộn, không có tính logic, hệ thống. Một số sinh viên, giáo viên hướng dẫn phải sửa chữa, yêu cầu làm lại 3 đến 4 lần mới đạt yêu cầu.

Sinh viên chưa nhận thức được tầm quan trọng của nghiên cứu khoa học, chưa thấy được lợi ích thiết thực của nó đối với hoạt động học tập, một số còn cho rằng, nghiên cứu khoa học là nhiệm vụ của thầy cô và của sinh viên khá, giỏi; việc nghiên cứu khoa học mất nhiều thời gian, công sức, đôi khi không thiết thực, hiệu quả, ảnh hưởng đến thời gian học tập.

Giảng viên hướng dẫn vừa đóng vai trò người hỗ trợ, vừa đóng vai trò chỗ dựa về tinh thần, tạo cảm hứng cho sinh viên. Nhưng thực tế cho thấy hiện nay một số giảng viên chưa thật sự nhiệt huyết với hoạt động nghiên cứu khoa học, việc thực hiện nhiệm vụ 
khoa học còn mang nặng tâm lý làm cho xong nhiệm vụ, một số giảng viên kỹ năng nghiên cứu khoa học còn hạn chế, một số giảng viên trình độ cao, có kỹ năng nghiên cứu khoa học lại phải kiêm nhiệm nhiều chức vụ, lên lớp nhiều (do bộ môn thiếu giảng viên). Một số giảng viên chưa phát huy được tính tích cực, chủ động của sinh viên trong học tập, chưa tin tưởng giao bài tập lớn, bài tiểu luận môn học cho sinh viên, chưa khuyến khích, khơi gợi được hứng thú nghiên cứu khoa học của các em.

Khó khăn về tài chính và các phương tiện hỗ trợ hoạt động nghiên cứu khoa học. Vấn đề tài chính luôn là bài toán nan giải đối với việc nghiên cứu khoa học, thực tế cho thấy có rất nhiều các chi phí như: Chi phí cho việc tìm kiếm (mua dữ liệu), chi phí cho mua nguyên vật liệu, công cụ, dụng cụ chạy thử, chi phí cho in ấn, chi phí cho điều tra khảo sát, chi phí thông tin liên lạc... Hầu hết các khoản chi phí cho hoạt động nghiên cứu khoa học sinh viên phải tự lo, vì vậy các em gặp rất nhiều khó khăn. Cũng có một số đề tài do tài chính eo hẹp nên phải lược bớt một số khảo sát, dẫn đến điều tra thiếu khách quan, kết quả của đề tài mức độ tin cậy chưa cao.

Nhiệm vụ chính của sinh viên là học tập, lịch học của từng học kỳ thường dao động từ 6 đến 8 môn, do đó việc phân chia thời gian cho học tập và cho nghiên cứu khoa học càng khó khăn. Thực tế cho thấy đa số các sinh viên năm cuối mới tương đối đủ kiến thức và nhận thức được ý nghĩa của việc nghiên cứu khoa học, nhưng áp lực về việc phải đi thực tập cuối khóa, lo tìm kiếm công việc trước khi ra trường làm cho họ không đủ thời gian để dành cho nghiên cứu khoa học.

\subsection{Giải pháp nâng cao hiệu quả hoạt động nghiên cứu khoa học của sinh viên}

Trên cơ sở thực trạng nêu trên, tôi mạnh dạn đề xuất một số giải pháp nhằm góp phần nâng cao hiệu quả hoạt động nghiên cứu khoa học của sinh viên trường Đại học Tân Trào.

\subsection{1. Đối với nhà trường}

- Điều chỉnh phân công chuyên môn hợp lý, học phần Phương pháp nghiên cứu khoa học giáo dục nên đưa vào nội dung học tập bắt buộc ngay năm đầu tiên để tất cả các sinh viên đều được trang bị những kiến thức, kĩ năng cần thiết đối với hoạt động này.

- Tổ chức các Hội thảo khoa học cấp khoa, cấp trường, các buổi tọa đàm, tập huấn...tạo cơ hội, môi trường cho sinh viên nghiên cứu, trao đổi với thầy cô, bạn bè.
- Có các chế độ chính sách khuyến khích sinh viên nghiên cứu khoa học, làm đồ án, khóa luận tốt nghiệp (cộng điểm học tập, rèn luyện, hỗ trợ kinh phí...)

- Tăng cường các điều kiện phục vụ hoạt động nghiên cứu khoa học của sinh viên: đầu tư hệ thống thông tin thư viện, phương tiện kỹ thuật hiện đại để hỗ trợ cho sinh viên trong việc tìm kiếm, thu thập tài liệu, thông tin.

- Xây dựng số tay nghiên cứu khoa học dành cho sinh viên.

- Gắn kết hoạt động NCKH của sinh viên với nhu cầu thực tế địa phương, các tổ chức và doanh nghiệp. Nhà trường cần chủ động và tăng cường xây dựng, duy trì và phát triển mối liên kết với các cơ quan, doanh nghiệp, các tổ chức giáo dục để gắn kết các hoạt động NCKH của sinh viên với nhu cầu thực tiễn. Tranh thủ tốt hơn sự quan tâm, tạo điều kiện của các cấp, các ngành và sự hỗ trợ của các tổ chức, cơ quan, doanh nghiệp đối với các sản phẩm, các đề tài nghiên cứu khoa học mà sinh viên thực hiện.

\subsection{2. Đối với giảng viên}

- Trong công tác giảng dạy nói chung và giảng dạy học phần Phương pháp nghiên cứu khoa học nói riêng, giảng viên phải luôn coi trọng việc tự học, tự nghiên cứu của sinh viên; phải lấy việc dạy kiến thức để dạy phương pháp chứ không chỉ thuần túy là cung cấp tri thức. Phương pháp dạy học phải từng bước làm cho phương pháp học tập của sinh viên ngày càng thống nhất với phương pháp nghiên cứu khoa học.

- Bản thân các giảng viên cần tích cực tham gia hoạt động nghiên cứu khoa học để sinh viên học tập, noi theo.

- Thường xuyên giao các bài tập lớn, tiểu luận môn học cho sinh viên, có thể thay thế điểm bài tiểu luận cho điểm thi học phần.

- Khi hướng dẫn sinh viên nghiên cứu khoa học, giảng viên cần chú ý phát huy tính chủ động, độc lập, sáng tạo của các em, tránh làm thay. Phải tôn trọng sự đa dạng của các ý kiến/quan điểm khi tìm kiếm giải pháp cho một vấn đề, khen gợi những ý kiến đúng, tư duy logic tốt. Thầy/cô cần định hướng cho sinh viên lựa chọn những đề tài phù hợp với thực tiễn, trình độ kiến thức và phù hợp với ngành nghề được đào tạo.

\subsection{3. Đối với Đoàn thanh niên, Hội sinh viên}

- Phát động phong trào nghiên cứu khoa học trong đoàn viên - sinh viên nhằm giúp sinh viên trao đổi, chia sẻ kinh nghiệm học tập, nghiên cứu khoa học. 
- Tổ chức nhiều cuộc thi, sân chơi trí tuệ nhằm thu hút sinh viên tham gia, chia sẻ, học hỏi kinh nghiệm, kiến thức. Thành lập và duy trì hiệu quả các câu lạc bộ học thuật dành cho sinh viên. Tăng cường tổ chức các buổi hội thảo, tọa đàm, giao lưu giữa sinh viên với những người thành công trong học tập, nghiên cứu khoa học, từ đó thắp sáng ước mơ, hoài bão trong sinh viên.

- Hội Sinh viên cần có những hoạt động tuyên truyền, đưa các thông tin về nghiên cứu khoa học đến với sinh viên, làm cho mỗi sinh viên tự ý thức được tầm quan trọng của hoạt động nghiên cứu khoa học và có nhu cầu nghiên cứu khoa học. Hội Sinh viên cần là cầu nối giữa sinh viên và nhà trường, các doanh nghiệp, trung tâm nghiên cứu.

\subsection{4. Đối với sinh viên}

- Cần nhận thức được ý nghĩa, tầm quan trọng của hoạt động nghiên cứu khoa học đối với bản thân, chuyển hoá được các yêu cầu, nhiệm vụ nghiên cứu khoa học của nhà trường, xã hội thành nhu cầu nghiên cứu của bản thân, từ đó mạnh dạn, tích cực, chủ động tham gia các hoạt động nghiên cứu khoa học do khoa, nhà trường tổ chức.

- Mỗi sinh viên cần tích cực, chủ động hơn nữa trong việc xây dựng kế hoạch học tập và nghiên cứu, xác định mục tiêu rõ ràng, tìm hiểu và lựa chọn cho mình phương pháp tự học, tự nghiên cứu hiệu quả, phù hợp.

\section{Kết luận}

Nghiên cứu khoa học trong sinh viên có ý nghĩa vô cùng to lớn, giúp cho sinh viên tiếp nhận cách học mới, hình thành phương pháp tự học, tự nghiên cứu, tập làm quen với hoạt động nghiên cứu khoa học căn bản, rèn luyện và nâng cao khả năng tư duy, tự khám phá, tự bồi dưỡng kiến thức. Không những thế, hoạt động nghiên cứu khoa học của sinh viên còn là một tiêu chí quan trọng đánh giá, xếp hạng chất lượng trường đại học. Thông qua bài báo này, tác giả mong muốn các bạn sinh viên nhận thức được ý nghĩa của việc nghiên cứu khoa học, coi nghiên cứu khoa học là một nhiệm vụ quan trọng song hành cùng hoạt động học tập khi ngồi trên ghế giảng đường đại học, có nhiều ý tưởng mới và có nhu cầu biến những ý tưởng khoa học trở thành hiện thực sinh động.

\section{TÀI LIỆU THAM KHẢO}

1. Vũ Cao Đàm, Phuơng pháp luận nghiên cúu khoa học, Nxb Giáo dục, Hà Nội, 2017;

2. Phó Đức Hòa, Phuong pháp nghiên cưu khoa học giáo duc Tiểu học, Nxb Đại học sư phạm, Hà Nội, 2009;

3. TS. Võ Thị Ngọc Lan - PGS. TS. Nguyễn Văn Tuấn, Phuoong pháp nghiên cúu khoa học giáo dục, Nxb Đại học Quốc gia TP Hồ Chí Minh, 2015;

4. PGS.TS. Phạm Viết Vượng, Phuơng pháp nghiên cúu khoa học giáo duc, Nxb Giáo dục, Hà Nội, 2001;

5. Luật Giáo dục Đại học (được Quốc hội nuớc Cộng hòa xã hội chủ nghĩa Việt Nam khóa XIII, kỳ họp thứ 3 thông qua ngày 18 tháng 6 năm 2012);

6. Viện hàn lâm khoa học xã hội Việt Nam, Bách khoa toàn thu Việt Nam, tại http://bachkhoatoanthu.vass.gov.vn, truy cập ngày 12/9/2017.

\section{Enhancing effectiveness of scientific research activities of students at Tan Trao University}

\section{Le Thi Thu Ha}

\section{Article info}

Recieved:

20/12/2017

Accepted:

$10 / 3 / 2018$

Keywords:

Science; scientific research; self-study; the scientificmethod of research.

\begin{abstract}
Scientific research has been identified as one of the focuses of Tan Trao University. Therefore, the University has always encouraged the lecturers and the students taken part in scientific research over the past years. However, the number of students taking part in scientific research is limited, the quality of the research is limited. In this article, the writer concentrated on the study of the actual situation of Tan Trao University students' scientific research. On the basis of, we propose some solutions to improve the efficiency of students' scientific research activities.
\end{abstract}

